PHYSICAL REVIEW D 94, 049905(E) (2016)

\title{
Publisher's Note: Transition radiation at radio frequencies from ultrahigh-energy neutrino-induced showers [Phys. Rev. D 93, 043010 (2016)]
}

Pavel Motloch, Jaime Alvarez-Muñiz, Paolo Privitera, and Enrique Zas (Received 28 July 2016; published 12 August 2016)

DOI: 10.1103/PhysRevD.94.049905

This paper was published online on 18 February 2016 with an error in Eq. (12). Equation (12) should read as

$$
\langle E \times R\rangle_{1 \mathrm{EeV}}^{\max }=1.52 \mathrm{mV} / \mathrm{MHz} \approx 2400\langle E \times R\rangle_{100 \mathrm{TeV}}^{\max } .
$$

The equation has been corrected as of 5 August 2016. The equation is incorrect in the printed version of the journal. 